\title{
Lumbar disc height and vertebral Hounsfield units: association with interbody cage subsidence
}

\author{
Alfred J. Pisano, MD, Donald R. Fredericks Jr., MD, Theodore Steelman, MD, Cory Riccio, MD, \\ Melvin D. Helgeson, MD, and Scott C. Wagner, MD
}

Department of Orthopaedics, Walter Reed National Military Medical Center, Bethesda, Maryland

\begin{abstract}
OBJECTIVE Postoperative subsidence of transforaminal lumbar interbody fusion (TLIF) cages can result in loss of lordosis and foraminal height, and potential recurrence of nerve root impingement. The objectives of this study were to determine factors associated with TLIF cage subsidence. Specifically, the authors sought to determine if preoperative disc height compared to cage height could be used to predict TLIF interbody cage subsidence, and if decreased postoperative vertebral Hounsfield units (HUs) predisposed to cage subsidence.
\end{abstract}

METHODS The authors retrospectively reviewed all patients undergoing instrumented TLIF from two institutions between July 2004 and June 2014. The preoperative disc height was measured for the operative and adjacent-level disc on MRI. The difference between cage and disc heights was measured and compared between the subsidence and nonsubsidence groups. The average HUs of the L1 vertebral body were measured on CT scans.

RESULTS Eighty-nine patients were identified with complete imaging and follow-up information. Forty-five patients $(50.6 \%)$ had evidence of interbody cage subsidence on follow-up CT. The average cage subsidence was $5.5 \mathrm{~mm}$ (range 2.2-10.8 mm). The average implant height was significantly higher in the subsidence group compared to the nonsubsidence group (12.6 vs $11.2 \mathrm{~mm}$ ). Additionally, the difference between cage height and preoperative adjacent-level disc height was also significantly larger in the subsidence group (3.8 vs $1.2 \mathrm{~mm}$ ). First lumbar vertebral body (L1) HUs were significantly higher in the nonsubsidence versus the subsidence group (167.8 vs $137.71 \mathrm{HUs}, p=0.002$ ). Multivariate logistic regression analysis identified suprajacent disc height and L1 HUs to be independent predictors of interbody cage subsidence. Receiver operating characteristic curves identified a suprajacent to cage height difference $>1.3 \mathrm{~mm}$ to have a $93.3 \%$ sensitivity for cage subsidence.

CONCLUSIONS This study is the first of its kind to demonstrate the association between vertebral body HUs and suprajacent disc height with the development of interbody cage subsidence after TLIF. The authors found that patients with lower HUs in the L1 vertebral body were more likely to experience subsidence, regardless of surgical level. Additionally, the study demonstrated that interbody cage height $>1.3 \mathrm{~mm}$ above the height of the suprajacent level is an independent risk factor for cage subsidence, with $93.3 \%$ sensitivity. These findings suggest that these factors may be utilized to create a template preoperatively for intraoperative cage selection.

https://thejns.org/doi/abs/10.3171/2020.4.FOCUS20286

KEYWORDS osteoporosis; cage subsidence; interbody fusion; cage templating; disc height; adjacent level

$\mathrm{T}$ RANSFORAMINAL lumbar interbody fusion (TLIF) was first described as an alternative to posterior interbody fusion in 1982 by Harms and Rolinger, utilizing a titanium mesh cage packed with bone graft material placed into the disc space via a transforaminal route, providing anterior column support and theoretically increasing the fusion surface area. ${ }^{2}$ The increased popularity of the TLIF procedure has led to numerous studies examining short- and long-term clinical and radiographic results, which have generally been reported as excellent. ${ }^{3-5}$ However, postoperative subsidence of the interbody device can result in loss of lordosis and foraminal height, and a theoretical risk of recurrence of nerve root impingement and radicular symptoms. Various clinical and biomechanical studies have evaluated the effects of different cage materials, sizes/shapes, bone mineral density (BMD), and cage position on the occurrence of subsidence. ${ }^{6-10}$

Additionally, the increasing prevalence of osteoporo-

ABBREVIATIONS BMD = bone mineral density; $B M P=$ bone morphogenetic protein; $H U$ = Hounsfield unit; $P E E K=$ polyetheretherketone; ROC = receiver operating characteristic; TLIF = transforaminal lumbar interbody fusion.

SUBMITTED April 3, 2020. ACCEPTED April 29, 2020.

INCLUDE WHEN CITING DOI: 10.3171/2020.4.FOCUS20286. 


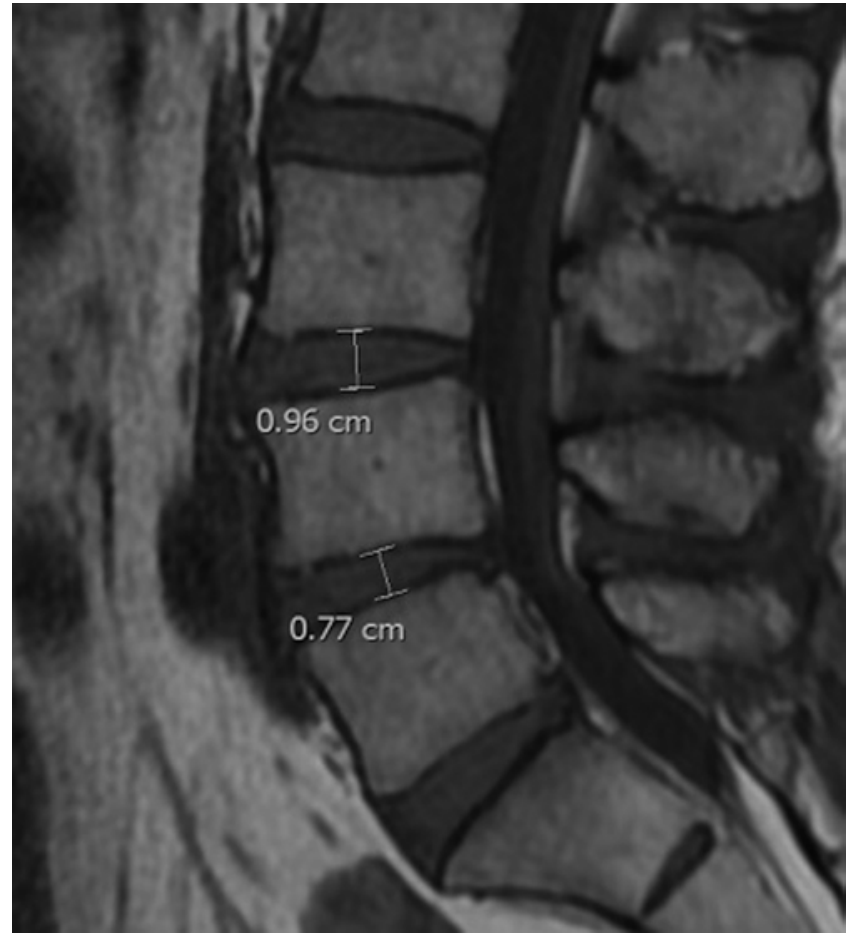

FIG. 1. Preoperative sagittal MRI disc height measurements. Operative disc height $7.7 \mathrm{~mm}$, suprajacent disc height $9.6 \mathrm{~mm}$; $14-\mathrm{mm}$ cage implanted. Evidence of cage subsidence is noted in Fig. 2.

sis and low BMD within the patient population presents unique clinical challenges for treating spine surgeons, and vertebral body Hounsfield units (HUs) have been proposed as an opportunistic surrogate for traditional BMD measurement techniques. ${ }^{11-13}$ However, to our knowledge, no studies have evaluated the relationship between preoperative disc heights of the affected and suprajacent levels, HU measurements of a single standardized lumbar vertebral body, and postoperative cage subsidence rates. We hypothesized that a relationship exists between preoperative disc height, first lumbar vertebral body (L1) $\mathrm{HU}$ measurements, and cage subsidence. We attempted to determine if preoperative disc heights can be utilized to create a template for the size of implanted TLIF cages to minimize subsidence rates, and if standardized measurements of lumbar vertebral HUs correlate with increased subsidence rates.

\section{Methods}

\section{Study Population and Criteria}

After IRB approval, we retrospectively reviewed all patients aged 50 years or older who underwent instrumented TLIF from two military institutions (Walter Reed National Military Medical Center and Fort Belvoir Community Hospital) between July 2004 and June 2014. Only patients with the following imaging were included in the study: preoperative MRI within 6 months of surgery, immediate postoperative radiographs, and minimum 12-month follow-up radiographs. Additionally, patients with routine postoperative CT scans within 6 months of surgery were

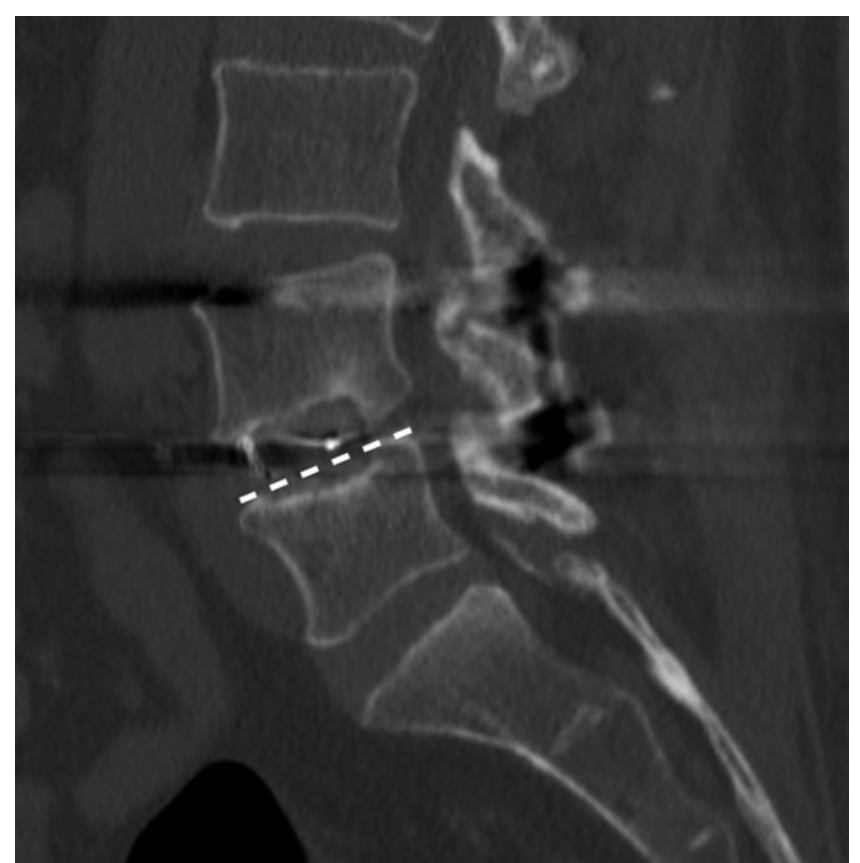

FIG. 2. Postoperative cage subsidence (dashed line) at the L4-5 level on sagittal CT.

also identified within the TLIF cohort. Patients undergoing multilevel TLIF or revision surgery were included in the study. We recorded demographic information and bone morphogenetic protein (BMP) use. Final fusion status was evaluated on final 12-month radiographs and recorded for each patient. Lastly, a chart review was performed to determine recurrence or persistence of symptoms.

\section{Data Collection}

We measured the central disc height at both the operative and suprajacent level on preoperative MRI (Fig. 1). In the case of multilevel surgery, the cephalad-most level was used as the operative level measurement. Disc height was measured on the T1-weighted midsagittal image at the midway point of the vertebral bodies. We then obtained all cage heights and types from operative reports. Finally, we identified cages that subsided using postoperative CT scans (Fig. 2). Subsidence was defined as greater than $2 \mathrm{~mm}$ of cage protrusion through the cephalad or caudal (or both) vertebral endplates. We then calculated the difference in cage height and the preoperative disc heights at both the operative and suprajacent levels in each patient.

CT HU measurements of L1 were recorded in all patients with routinely obtained CT scans within 6 months of surgery, utilizing a previously described technique. ${ }^{14,15}$ The first lumbar segment was chosen as an opportunistic BMD measurement level due to its nonroutine use in instrumented TLIF procedures, lack of suspected degenerative changes, and the prevalence of level inclusion in chest, abdominal, lumbar, and pelvic CT scans. HU values were calculated on postoperative CT scans by using axial, sagittal, and coronal circles of best fit of L1. 
TABLE 1. Demographic data

\begin{tabular}{lcccc}
\hline \multicolumn{1}{c}{ Variable } & $\begin{array}{c}\text { Total } \\
(\%)\end{array}$ & $\begin{array}{c}\text { Subsidence } \\
(\%)\end{array}$ & $\begin{array}{c}\text { Subsidence } \\
(\%)\end{array}$ & $\begin{array}{c}\mathrm{p} \\
\text { Value }\end{array}$ \\
\hline No. of patients & 89 & $45(51)$ & $44(49)$ & \\
\hline Males & $53(60)$ & & & \\
\hline Average age, yrs & 59.9 & 60.2 & 58.1 & 0.17 \\
\hline $\begin{array}{l}\text { Average no. of } \\
\text { levels fused }\end{array}$ & & 2.0 & 1.7 & 0.06 \\
\hline $\begin{array}{l}\text { Average implant } \\
\text { length, mm }\end{array}$ & & 21.4 & 22.3 & 0.10 \\
\hline L4 mean HUs, BMD & & 149.8 & 160.8 & 0.20 \\
\hline
\end{tabular}

\section{Statistical Analysis}

Means of differences were compared between the subsidence and nonsubsidence groups. We used the MannWhitney U-test to determine statistical significance of means between the two groups. The chi-square test was used to compare proportions, where applicable. Multivariate logistic regression was utilized to test for independent associations between variables. Receiver operating characteristic (ROC) curves were calculated and used to ascertain height differential thresholds for subsidence; $p$ values $\leq 0.05$ were considered statistically significant.

\section{Results}

We identified 89 patients with complete imaging and follow-up information. The cohort was $59.6 \%$ male and the average age was 59.9 years. Forty-five patients (50.6\%) had evidence of interbody cage subsidence on follow-up CT scans. Patients had an average clinical follow-up of 27 months. No significant differences in preoperative demographics were noted (Table 1). BMP use was reported in 30 patients, and there was no increased rate of subsidence between groups based on BMP use (50\% vs $50.8 \%$, p > $0.05)$. The vast majority of interspace implants (97\%) were polyetheretherketone (PEEK) bullet-shaped cages. The average cage subsidence was $5.5 \mathrm{~mm}$ (range $2.2-10.8 \mathrm{~mm}$ ).

The average preoperative disc height for the operative levels and the immediately suprajacent levels was $7.1 \mathrm{~mm}$ and $9.3 \mathrm{~mm}$ for all patients, which was statistically significantly different $(\mathrm{p}<0.0001)$. This finding remained true within both the subsidence and nonsubsidence groups (nonsubsidence group: 7.1 vs $9.9 \mathrm{~mm}, \mathrm{p}<0.0001$; subsidence group: 7.1 vs $8.8 \mathrm{~mm}, \mathrm{p}=0.001$ ). The average suprajacent disc height was significantly larger in the nonsubsidence group ( $9.9 \mathrm{vs} 8.8 \mathrm{~mm}, \mathrm{p}=0.02$ ). The preoperative disc height at the surgical levels was not significantly different between the subsidence and nonsubsidence groups (7.1 vs $7.1 \mathrm{~mm}, \mathrm{p}>0.05$ ).

The average cage height in the subsidence group was significantly larger than in the nonsubsidence group (12.6 vs $11.2 \mathrm{~mm}, \mathrm{p}<0.001$ ). Additionally, the percentage of patients with an implant height greater than the median $(12 \mathrm{~mm})$ was significantly higher in the subsidence group ( $42.2 \%$ vs $20.5 \%, p=0.03)$. The height difference between cage and operative level disc was significantly larger in the
TABLE 2. Height differences in subsidence and nonsubsidence groups

\begin{tabular}{lccc}
\hline & Subsidence & $\begin{array}{c}\text { No } \\
\text { Subsidence }\end{array}$ & $\begin{array}{c}\mathrm{p} \\
\text { Value }\end{array}$ \\
\hline Mean cage height, $\mathrm{mm}$ & 12.6 & 11.2 & $<0.001$ \\
\hline $\begin{array}{l}\text { Cage to operative level } \\
\text { height, mm }\end{array}$ & 5.5 & 3.9 & 0.01 \\
\hline $\begin{array}{l}\text { Cage to suprajacent } \\
\text { level height, mm }\end{array}$ & 3.8 & 1.2 & $<0.0001$ \\
\hline
\end{tabular}

subsidence group compared to the nonsubsidence group (5.5 vs $3.9 \mathrm{~mm}, \mathrm{p}=0.01$ ). Similarly, the subsidence group had a significantly larger height difference between cage and suprajacent level disc in comparison to the nonsubsidence group (3.8 vs $1.2 \mathrm{~mm}, \mathrm{p}<0.0001$; Table 2). A representative example of initial disc height, immediately postoperative and at final follow-up, that demonstrates the discordance between initial disc height, cage height, and subsidence is shown in Fig. 3.

We performed ROC curves to compare the difference between cage height and disc height at the surgical and suprajacent levels (Figs. 4 and 5). The ROC curve for operative disc height differential followed a relatively linear pattern, while the curve for suprajacent level height differential followed a more predictable ROC curve pattern. A height difference at the suprajacent level of 1.3 $\mathrm{mm}$ yielded $93.3 \%$ sensitivity for developing subsidence, with a false-positive rate of 53\%. For the operative level, a height difference of $2.1 \mathrm{~mm}$ also yielded a sensitivity of $93.3 \%$ for subsidence (false-positive rate $77.0 \%$ ). Utilizing height differential cutoffs of $3.6 \mathrm{~mm}$ and $5.5 \mathrm{~mm}$ for the suprajacent and operative levels, respectively, would yield sensitivities of $57 \%$ and $51.1 \%$ for subsidence, but with much lower false-positive rates $(18.8 \%$ and $27 \%$, respectively). Similarly, we found that a greater than $3.0-\mathrm{mm}$ cage to suprajacent level differential threshold yielded a significant odds ratio of 3.9 (95\% CI 1.61-9.37) for subsidence. We failed to identify a difference in the rates of fusion between the subsidence and nonsubsidence groups ( $82 \%$ vs $93 \%, \mathrm{p}=0.08)$. We did not find any statistically significant differences in the percentage of patients reporting persistence or recurrence of radiculopathy between the groups at final follow-up (47\% [subsidence] vs 38\% [nonsubsidence], $\mathrm{p}=0.383$ ).

We compared subsidence and nonsubsidence HU measurements. First lumbar vertebral body (L1) HU measurements were significantly higher in the nonsubsidence versus subsidence group in all measured planes: coronal $(170.69 \pm 15.61$ vs $138.64 \pm 14.21$ HUs, $p=0.003)$, sagittal (175.14 \pm 17.26 vs $140.16 \pm 13.71$ HUs, $\mathrm{p}=0.002)$, axial HUs $(157.57 \pm 12.26$ vs $134.35 \pm 12.30 .83$ HUs, $p=0.009)$, and composite HUs $(167.8 \pm 14.04$ vs $137.71 \pm 12.83$ HUs, $\mathrm{p}=0.002$ ). A total of 17 patients had average L1 HU values $<110$, which is a previously defined cutoff value for osteoporosis; ${ }^{12} 70.6 \%$ of patients in that group showed evidence of subsidence, while only $45.8 \%$ of patients with average HUs $>110$ showed subsidence $(\mathrm{p}=0.06)$.

Multivariate logistic regression identified cage to su- 

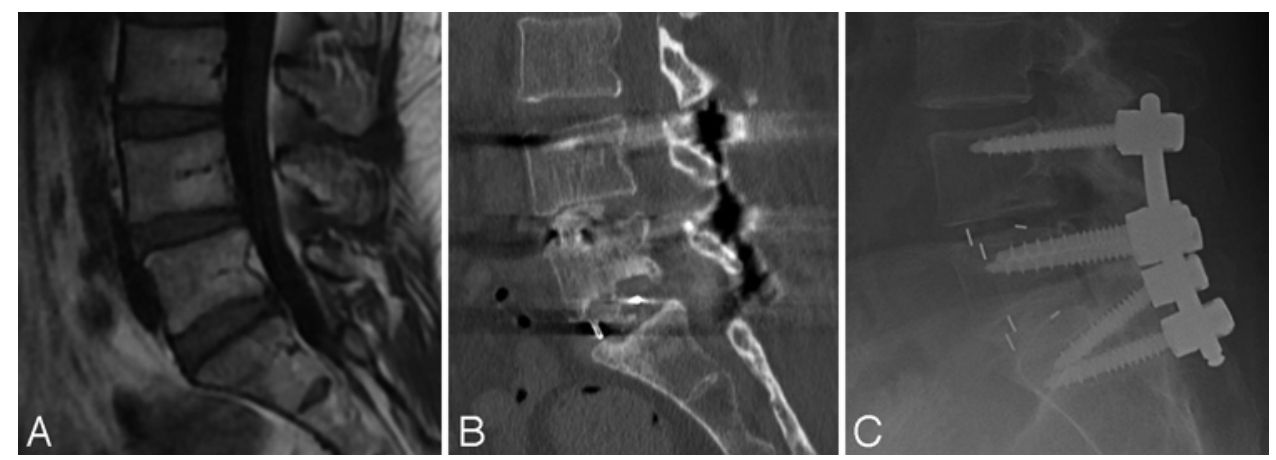

FIG. 3. Preoperative sagittal MR image (A), 6-month postoperative sagittal CT scan (B), and 6-month postoperative lateral plain radiograph (C) obtained in a patient with multilevel evidence of subsidence.

prajacent disc height difference and average L1 vertebral body HUs to be independent, significant predictors of cage subsidence ( $\mathrm{p}=0.01$ and 0.008 , respectively). All other variables, including the number of levels treated, cage height, patient BMI, BMP use, age, and sex, were not shown to be independently significant.

\section{Discussion}

TLIF, when utilized in a short-segment construct, is typically indicated for instability and radiculopathy. In addition to the anterior column support and large surface area for $360^{\circ}$ fusion, the indirect decompression afforded by the increase in foraminal height is also effective in the treatment of radiculopathy. If the interbody device subsides, foraminal height is lost, potentially leading to a recurrence or persistence of symptoms. We found an overall subsidence rate of $44 \%$, consistent with other published data; Lee et al. found a subsidence rate of $38 \%$ in a series of 21 patients with 2 -year follow-up. ${ }^{16}$ Increased implant height was found to be a risk factor for subsidence in our series, with a significant difference between the subsidence and nonsubsidence groups of $1.4 \mathrm{~mm}$ (12.6 vs 11.2 $\mathrm{mm}, \mathrm{p}<0.001)$, which is in contradistinction to several prior studies showing this factor to have no impact on subsidence. $7,16,17$

In our study, multivariate logistic regression identified the height difference between the suprajacent disc height

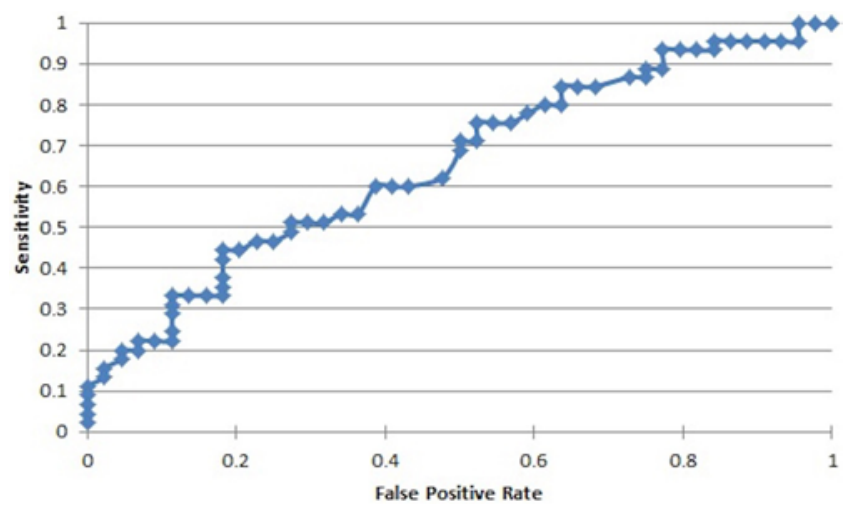

FIG. 4. ROC curve of operative disc height and cage height difference. and interbody cage (measured on non-weightbearing $\mathrm{MRI}^{18}$ ), as well as average L1 vertebral body HUs, to be independent significant predictors of interbody cage subsidence after TLIF ( $\mathrm{p}=0.01$ and 0.008 , respectively). As our results suggest, an interbody cage that is larger than the suprajacent (nonsurgical) disc space may be prone to subsidence. The subsidence group had a significantly larger discrepancy between the suprajacent level disc height and implanted cage height, as compared to the nonsubsidence group ( 3.8 vs $1.2 \mathrm{~mm}, \mathrm{p}<0.0001)$. An ROC curve calculated for the suprajacent level height difference yielded a threshold of $1.3 \mathrm{~mm}$ for $93.3 \%$ sensitivity for subsidence, or $3.6 \mathrm{~mm}$ for an $18.9 \%$ false-positive rate. Our results also demonstrate that suprajacent level disc height may be a reliable adjunct for templating cages, as the level being treated is often collapsed or asymmetrical, making height estimation and comparisons more difficult. In the subsidence group, the measured height discrepancy between operative level disc height and implanted cage was significantly larger (5.5 vs $3.9 \mathrm{~mm}, \mathrm{p}=0.01$ ). The ROC curve for the operative level height yielded $93.3 \%$ sensitivity for subsidence with a height differential threshold of $2.1 \mathrm{~mm}$, or $5.5 \mathrm{~mm}$ with a low false-positive rate $(27.0 \%)$, but this association followed a more linear ROC pattern. On multivariate analysis, the operative level disc height was not found to be independently associated with cage subsidence.

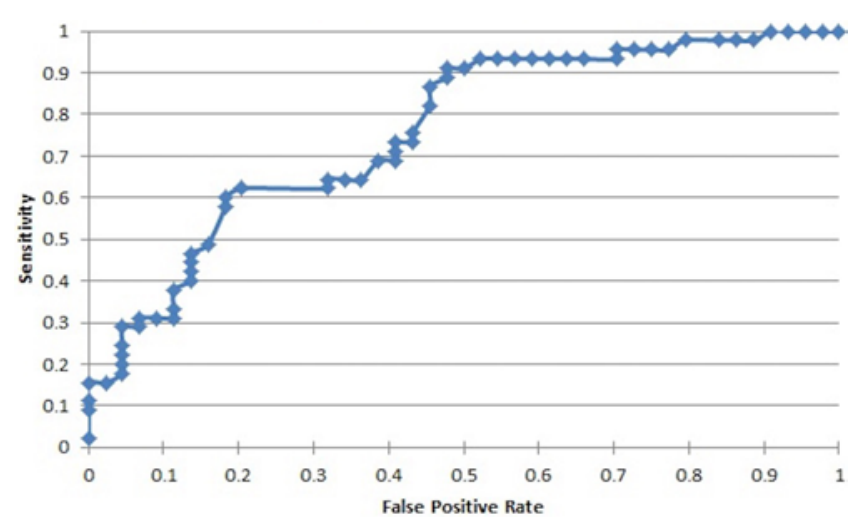

FIG. 5. ROC curve of suprajacent disc height and cage height difference. 
Ultimately, our results suggest that placing a larger interbody device into a smaller disc space may predispose toward subsidence, and we hypothesize that utilization of the preoperative suprajacent disc height as a sizing template may more appropriately reflect the ideal cage size, and potentially minimize the incidence of subsidence. We theorize that increased differential between cage and disc height may ultimately lead to violation of the endplate over time, due to increased contact pressure and poor force distribution..$^{19}$ It is important to note, however, that the vast majority of the interbody cages used in this study were PEEK bullet cages, which have a large endplate contact surface area within the central portion of the disc space, rather than anterior/edge-loading crescent cages, and so our findings are specific to this type of TLIF interbody cage with regard to subsidence risk.

Furthermore, we found that lower average composite HU measurements of L1 were independently associated with an increased risk of cage subsidence. The patients without evidence of cage subsidence had significantly higher HUs compared to patients with subsidence (167.8 \pm 14.04 HUs vs $137.71 \pm 12.83$ HUs, respectively; $p=0.002$ ). These findings suggest that a relationship exists between lower HUs and the likelihood of cage subsidence after TLIF, despite these measurements being performed on a level not included in the surgical construct. Multiple studies have validated the correlation between HUs as measured on CT and BMD on dual-energy x-ray absorptiometry to determine bone quality and fracture risk, as well as the utility of HU measurement as an opportunistic surrogate for quantitative BMD assessment. ${ }^{12-15,20-22}$ Hendrickson et al. reported excellent reproducibility and accuracy in identifying osteoporosis of the spine using CT attenuation measures such as HUs. ${ }^{11}$ Our study demonstrated a significant trend of lower lumbar vertebral body HUs in the subsidence group, and corroborates the work of several studies demonstrating low BMD to be a risk factor for subsidence. ${ }^{17,19}$ Pickhardt et al. demonstrated L1 thresholds of 135 HUs for balanced sensitivity and specificity, and a threshold of 110 HUs for $90 \%$ specificity for osteoporosis, ${ }^{15}$ which are similar numbers to those reported by Wagner et al..$^{20}$ Our series confirms several prior studies that failed to show any statistically significant correlation with subsidence and recurrence of preoperative symptoms. ${ }^{3,5}$ In reviewing 139 operative segments, Oh et al. found that subsidence did not correlate with worse clinical outcomes. ${ }^{6}$ The rate of fusion in this study is similar to the rate in the published literature. ${ }^{23-25}$ Additionally, we identified a trend toward a decreased fusion rate in the group with subsidence, although this finding was not statistically significant $(82 \%$ vs $93 \%, \mathrm{p}=0.08)$. A correlation between subsidence and pseudarthrosis has not been previously reported in the literature.

Our study was subject to several limitations. First, as with any retrospective cohort analysis, clinical data are limited by the accuracy of documentation. Furthermore, despite a minimum age of 50 years for inclusion in this study, our patients were still relatively young with an average age of 58.9 years, and future studies may consider evaluating older patients with greater BMD loss. Preoperative MRI was used to measured disc height, which may not be as accurate as CT; however, few patients in this cohort had preoperative CT scans for evaluation, while MRI was ubiquitous. We did not include patient-reported outcome measures in this study, and our primary clinical outcome was merely defined as persistence or recurrence of symptoms. Lastly, we did not establish the final location of the cage on the endplates, and cages placed more anteriorly or along the apophyseal ring are theoretically supported on denser bone. Future studies utilizing HUs to map the density of the lumbar vertebral bodies are ongoing. Interestingly, we were unable to show any association between BMP use and subsidence, which contradicts published literature on the topic. ${ }^{26-28}$ This finding highlights a limitation of our study, i.e., it is not clear from the operative reports whether BMP was placed within the disc space or in the posterolateral gutter. Because this study incorporates multiple surgeons at more than one institution, there was no rigorously standardized surgical technique, and our results must be interpreted within that context. Finally, many patients with degenerative lumbar conditions develop endplate sclerosis that, despite an actual diagnosis of central osteopenia or osteoporosis, may paradoxically be protected from subsidence; this statement is only a hypothesis, however, and has not been studied. Despite these limitations, we were able to demonstrate a positive correlation between lower vertebral HUs and the risk for subsidence, as well as validate the use of preoperative disc height on MRI for creating a cage template and planning the size of the implant.

\section{Conclusions}

We found a significant association between larger interbody devices and postoperative cage subsidence after TLIF. In addition, we found that the suprajacent level disc height may be used to accurately create a template the size of the planned interbody cage. Targeting an implant size similar to the preoperative template size may reduce the rate of postoperative cage subsidence after TLIF. Finally, our findings suggest that lower lumbar vertebral body HU measurements may be a risk factor for interbody cage subsidence.

\section{References}

1. Harms J, Rolinger H. A one-stager procedure in operative treatment of spondylolistheses: dorsal traction-reposition and anterior fusion (author's transl). Article in German. Z Orthop Ihre Grenzgeb. 1982;120(3):343-347.

2. Abd El-Kader HEl-B. Transforaminal lumbar interbody fusion for management of recurrent lumbar disc herniation. Asian Spine J. 2016;10(1):52-58.

3. Potter BK, Freedman BA, Verwiebe EG, et al. Transforaminal lumbar interbody fusion: clinical and radiographic results and complications in 100 consecutive patients. J Spinal Disord Tech. 2005;18(4):337-346.

4. Salehi SA, Tawk R, Ganju A, et al. Transforaminal lumbar interbody fusion: surgical technique and results in 24 patients. Neurosurgery. 2004;54(2):368-374.

5. Rosenberg WS, Mummaneni PV. Transforaminal lumbar interbody fusion: technique, complications, and early results. Neurosurgery. 2001;48(3):569-575.

6. Oh KW, Lee JH, Lee JH, et al. The correlation between cage subsidence, bone mineral density, and clinical results in pos- 
terior lumbar interbody fusion. Clin Spine Surg. 2017;30(6): E683-E689.

7. Kim MC, Chung HT, Cho JL, et al. Subsidence of polyetheretherketone cage after minimally invasive transforaminal lumbar interbody fusion. J Spinal Disord Tech. 2013;26(2): 87-92.

8. Nemoto O, Asazuma T, Yato Y, et al. Comparison of fusion rates following transforaminal lumbar interbody fusion using polyetheretherketone cages or titanium cages with transpedicular instrumentation. Eur Spine J. 2014;23(10):2150-2155.

9. Faizan A, Kiapour A, Kiapour AM, Goel VK. Biomechanical analysis of various footprints of transforaminal lumbar interbody fusion devices. J Spinal Disord Tech. 2014;27(4): E118-E127.

10. Tokuhashi Y, Ajiro Y, Umezawa N. Subsidence of metal interbody cage after posterior lumbar interbody fusion with pedicle screw fixation. Orthopedics. 2009;32(4). doi: 10.3928/01477447-20090401-18

11. Hendrickson NR, Pickhardt PJ, Del Rio AM, et al. Bone mineral density T-scores derived from CT attenuation numbers (Hounsfield units): clinical utility and correlation with dualenergy x-ray absorptiometry. Iowa Orthop J. 2018;38:25-31.

12. Schreiber JJ, Anderson PA, Rosas HG, et al. Hounsfield units for assessing bone mineral density and strength: a tool for osteoporosis management. J Bone Joint Surg Am. 2011;93(11): 1057-1063.

13. Wagner SC, Dworak TC, Grimm PD, et al. Measurement of distal ulnar Hounsfield units accurately predicts bone mineral density of the forearm. J Bone Joint Surg Am. 2017;99(8):e38.

14. Lee S, Chung CK, Oh SH, Park SB. Correlation between bone mineral density measured by dual-energy x-ray absorptiometry and Hounsfield units measured by diagnostic CT in lumbar spine. J Korean Neurosurg Soc. 2013;54(5):384-389.

15. Pickhardt PJ, Pooler BD, Lauder T, et al. Opportunistic screening for osteoporosis using abdominal computed tomography scans obtained for other indications. Ann Intern Med. 2013;158(8):588-595.

16. Lee N, Kim KN, Yi S, et al. Comparison of outcomes of anterior, posterior, and transforaminal lumbar interbody fusion surgery at a single lumbar level with degenerative spinal disease. World Neurosurg. 2017;101:216-226.

17. Beutler WJ, Peppelman WC Jr. Anterior lumbar fusion with paired BAK standard and paired BAK Proximity cages: subsidence incidence, subsidence factors, and clinical outcome. Spine J. 2003;3(4):289-293.

18. Cheung JP-Y, Shigematsu H, Cheung KM-C. Verification of measurements of lumbar spinal dimensions in T1- and T2weighted magnetic resonance imaging sequences. Spine $J$. 2014;14(8):1476-1483.

19. Tempel ZJ, Gandhoke GS, Okonkwo DO, Kanter AS. Impaired bone mineral density as a predictor of graft subsidence following minimally invasive transpsoas lateral lumbar interbody fusion. Eur Spine J. 2015;24(suppl 3):414-419.

20. Wagner SC, Formby PM, Helgeson MD, Kang DG. Diagnosing the undiagnosed: osteoporosis in patients undergoing lumbar fusion. Spine (Phila Pa 1976). 2016;41(21):E1279E1283.

21. Marinova M, Edon B, Wolter K, et al. Use of routine thoracic and abdominal computed tomography scans for assessing bone mineral density and detecting osteoporosis. Curr Med Res Opin. 2015;31(10):1871-1881.

22. Formby PM, Kang DG, Helgeson MD, Wagner SC. Clinical and radiographic outcomes of transforaminal lumbar interbody fusion in patients with osteoporosis. Global Spine J. 2016;6(7):660-664.
23. Peng CW, Yue WM, Poh SY, et al. Clinical and radiological outcomes of minimally invasive versus open transforaminal lumbar interbody fusion. Spine (Phila Pa 1976). 2009;34(13): 1385-1389.

24. Smith AJ, Arginteanu M, Moore F, et al. Increased incidence of cage migration and nonunion in instrumented transforaminal lumbar interbody fusion with bioabsorbable cages. $\mathrm{J} \mathrm{Neu}$ rosurg Spine. 2010;13(3):388-393.

25. Faundez AA, Schwender JD, Safriel Y, et al. Clinical and radiological outcome of anterior-posterior fusion versus transforaminal lumbar interbody fusion for symptomatic disc degeneration: a retrospective comparative study of 133 patients. Eur Spine J. 2009;18(2):203-211.

26. Helgeson MD, Lehman RA Jr, Patzkowski JC, et al. Adjacent vertebral body osteolysis with bone morphogenetic protein use in transforaminal lumbar interbody fusion. Spine J. 2011; 11(6):507-510.

27. Knox JB, Dai JM III, Orchowski J. Osteolysis in transforaminal lumbar interbody fusion with bone morphogenetic protein-2. Spine (Phila Pa 1976). 2011;36(8):672-676.

28. Vaidya R, Sethi A, Bartol S, et al. Complications in the use of rhBMP-2 in PEEK cages for interbody spinal fusions. $J$ Spinal Disord Tech. 2008;21(8):557-562.

\section{Disclaimer}

The views expressed in this manuscript are those of the authors and do not reflect the official policy of the Department of Navy, Department of Defense, or US Government. The authors are employees of the US government. This work was prepared as part of their official duties and as such, there is no copyright to be transferred.

\section{Disclosures}

The authors report no conflict of interest concerning the materials or methods used in this study or the findings specified in this paper.

\section{Author Contributions}

Conception and design: Fredericks, Pisano, Steelman, Riccio, Wagner. Acquisition of data: Fredericks, Pisano, Steelman, Wagner. Analysis and interpretation of data: Pisano, Riccio, Wagner. Drafting the article: Fredericks, Pisano, Steelman, Riccio. Critically revising the article: Fredericks, Steelman, Riccio, Helgeson, Wagner. Reviewed submitted version of manuscript: Helgeson. Statistical analysis: Wagner. Administrative/technical/material support: Pisano, Helgeson, Wagner. Study supervision: Pisano, Helgeson, Wagner.

\section{Supplemental Information}

\section{Previous Presentations}

Portions of this work were presented in abstract form as proceedings at the annual meeting of the North American Spine Society in Los Angeles, California, September 26-29, 2018.

\section{Correspondence}

Donald R. Fredericks Jr.: Walter Reed National Military Medical Center, Bethesda, MD. donaldfredericks.jr@gmail.com. 